\title{
Histopathological review of renal pelvic tumours of Balkan nephropathy cases from Southern Serbia
}

\author{
Diana Herman ${ }^{1}$, Goran Stanojević ${ }^{2}$, Nikola Pavlović ${ }^{3}$ and Peter Mantle ${ }^{4}$
}

Cite this article: Herman D, Stanojević G, Pavlović N and Mantle P: Histopathological review of renal pelvic tumours of Balkan nephropathy cases from Southern Serbia. Ann Urol Oncol 2020; 3(1): 15-21. https:// doi.org/10.32948/auo.2020.07.11

\begin{abstract}
Background Urothelial tumour frequently associates with atrophied kidney pelvis in Balkan endemic nephropathy but histopathology illustration lacks both definition and immune profile for differential diagnosis from renal cell carcinoma.

Methods Histology sections of anonymized archived renal tumours and associated kidney of eight local Balkan nephropathy patients were stained by haematoxylin and eosin, and immune profiles revealed by automated clinical immunohistochemistry, to refine carcinoma diagnosis and illustration.

Results Two low-grade and four high-grade papillary carcinomas were diagnosed. Two cases of high-grade urothelial carcinomas with solid architecture, invasive features and aggressive biological behaviour are also described with illustrated immune profiles.

Conclusion Refined pelvic tumour histopathology can now facilitate comparison across endemic areas within the Balkans and beyond. Notably, immune profiles of the present tumours correspond to those of contemporary urothelial cancers studied in Slovakia, where Balkan-like nephropathies have not been recognised. Some etiological considerations can be discussed.
\end{abstract}

Key words immunohistochemistry, papillary carcinoma, high grade urothelial carcinoma, elective nephrectomy

\footnotetext{
1. Pathology Department, County Hospital, Timisoara, Romania.

2. Pathology Department, County Hospital, Leskovac, Serbia.

3. University of Niš, Niš, Serbia.

4. Centre for Environmental Policy, Imperial College London, London UK.

Correspondence: Prof. Peter G. Mantle (Centre for Environmental Policy, Imperial College London, London UK.; Email: p.mantle@imperial.ac.uk).
} 


\section{Introduction}

The Balkan endemic nephropathy (BEN) is a bilateral renal atrophy which has been recognised in specific rural settlements in the last century, since first studied specifically in the Vratza district in Bulgaria and designated as a nosological entity in the early 1950s [1]. Forty-one villages were affected, 15 of them seriously, and during the decade after 1965 BEN mortality was over $40 \%$ for women and about $30 \%$ for men [2]. Hyperendemic areas were also defined in South-East Croatia, several parts of Bosnia and Serbia, and in South-Western Romania, although officially denied until 1990 after the revolution. The condition was expressed as a silent disease and life was extended after diagnosis by provision of haemodialysis. In some areas social provision of suitably nutritious meals for people with renal insufficiency was made, until funding expired following political changes. In Bulgaria it soon became apparent that there was a 90 -fold increased incidence of urothelial neoplasms in BEN subjects relative to that in nonendemic communities [2]. In former Yugoslavia a similar 100-fold differential was recognised $[3,4]$ but focus on the hyperendemic area near Slavonski Brod (now in Croatia) during 1974-1989 revealed a 5 -fold increased proportion in endemic areas relative to non-endemic areas. Notably, in endemic areas cases were younger patients, females were more severely affected, tumours were in the upper urinary tract as opposed to bladder, and lesions were frequently multiple and malignant instead of single and benign [5]. However, within an endemic area the differential rose to 14fold. In Slavonski Brod (1975-90) 193 urinary tract tumours were described as papillary and solid carcinomas of the transitional cell type, squamous-cell carcinomas and carcinomas in situ. BENassociated upper urinary tract tumours were designated papillary transitional cell carcinoma. Nevertheless the sparse published illustration remains uninformative. In spite of socio-political and economic changes, the disease still persists. Putative aetiological agents have multiplied over the years, including water polluted by the debris of wars or by leachate from open-cast coal mines. Correlation of peaks in death rates with exceptional rainfall on the predominantly flood-plain location of most BEN villages [6] suggested transmissible viruses, microbiological agents or their toxic metabolites. Isolation of a common foodstuff-spoilage Penicillium from the hyperendemic Romanian village of Erghevita and its toxicity to rats demonstrated in the UK [7] supported a fungal toxin hypothesis. Concurrent seasonal porcine nephropathy in the Danish bacon industry in the mid-1900s, for which the newly discovered nephrotoxin ochratoxin A was found to be the principal cause via moulded barley in feed, became a new focus of attention as an experimental rodent model for BEN. Although the bacon industry did not give time for expression of any urothelial carcinogenesis in pigs, its potent carcinogenesis in experimental rats and mice was demonstrated during lifetime exposure $[8,9]$.

Human renal disease involving urological tumours in a Belgian outbreak in the 1990s in middle-aged women was attributed to a herbal-based medication for slimming [10]. The principal aristolochic acid component, well known as a potential risk factor in oriental herbal medicaments, was shown to be the cause via accidental use of an Aristolochia component and has since been a major focus of research attention on BEN, assuming that it has similar causation. Considerable scientific literature has accumulated, cited in a recent open access review [11] as background experience for the present study of BEN-associated urothelial tumours. From the nearly 60 years of history above it could be expected that most of the renal pelvis tumours sourced for the present study in the context of BEN could be linked aetiologically to that renal disease, but not necessary all. We have recent experience in applying clinical immunohistochemistry (IHC) to review of experimental cancers in rats given protracted or lifetime exposure to ochratoxin A $[12,13]$. Refinement of histopathology, of value in offering new understanding of the origin and context of cancers, contrasts with literature illustration of BEN-associated tumours $[14,15]$ partly due to risks of postmortem tissue changes in subjects who most likely die at home. Thus we have studied archived urothelial tumours from elective nephrectomy in BEN subjects, selecting only those located in the renal pelvis. This potentially allows also immunohistochemical categorisation of any tumour which might have arisen from renal cortex or medulla and its origin obscured through subsequent distortions from the BEN characteristic of renal atrophy.

\section{Materials and methods}

Experimental material for renal pelvis tumours from BEN subjects was paraffin blocks deposited in pathology archives at the Leskovac hospital during the period 2007-2016. Histology sections $(3 \mu \mathrm{m})$ were mounted on glass slides, necessarily as anonymous and denatured tissue to remain outside UK import regulation for medical research by Imperial College London. For IHC, sections were mounted on charged slides (TOMO, Matsunami, Japan) and processed in the Cell Pathology Laboratory of South West London Pathology at St George's Hospital, Tooting, variously applying panels of antibodies (by Roche except where stated) in fully-automated BenchMark ULTRA immunohistochemistry processing as required to assist clinical diagnoses. The following antibodies (clone) were used: GATA3 (L50-823), PAX8 (Menarini, rabbit polyclonal), CD34 (Q BEND 10), p63 (4A4), CK AE1/ AE3 (Anti-Pan Keratin, Ventana PCK26), CK7 (SP52), CK20 (SP33), SMA (clone 1A4, Dako) and Vimentin (clone V9, Dako, Novocastra). Each antibody was provided by the supplier with its own data sheet, indicating the clone, concentration and working protocol. Haematoxylin \& Eosin staining was also performed for preliminary standard tissue differentiation of nuclear (blue) and cytoplasmic components (red). For IHC, after applying DAB chromogen, nuclei were counterstained blue with haematoxylin.

\section{Results}

Histopathological diagnoses for the pelvic tumours and a sample from the associated kidney. Figures' scale bar in bottom right-hand corner becomes evident with magnification.

Case 1: Urothelial papillary carcinoma, low grade, non-invasive (no muscle invasion). Intratumoural focal calcifications. Renal parenchyma: focal chronic inflammation, no significant fibrosis.

Case 2: Urothelial papillary carcinoma, low grade, non-invasive, with pushing border (Figure 1). IHC confirming urothelial origin: tumour cells are positive for GATA3, p63, CK7 (Figure 2) and negative for CK20. Renal parenchyma: foci of chronic inflammatory infiltrate, rare glomerulosclerosis and minimal fibrosis of the cortex and cortico-medullary junction.

Case 3: Foci of high grade urothelial papillary carcinoma with lamina propria invasion, on a background of low grade urothelial carcinoma; no muscle invasion. Renal parenchyma: diffuse chronic inflammatory infiltrate, mild fibrosis with focal enhancement.

Case 4: Urothelial papillary carcinoma, high grade, invasive (Figure 3). Tumour is intensely and diffusely CK7 positive; there is muscle invasion, as highlighted per SMA positive (Figure 4). Renal parenchyma: patchy lymphoid infiltrate, a few tubules with microcystic changes, focal arteriolar hyalinosis. Mild fibrosis, no glomerulosclerosis.

Case 5: Urothelial papillary carcinoma, high grade, with focal lamina propria invasion. CK20 is positive on a few tumour cells (Figure 5). Small renal tissue fragment present, with marked inflammatory infiltrate (lymphocytes and eosinophils), glomerulosclerosis, tubular destruction and minimal fibrosis. 


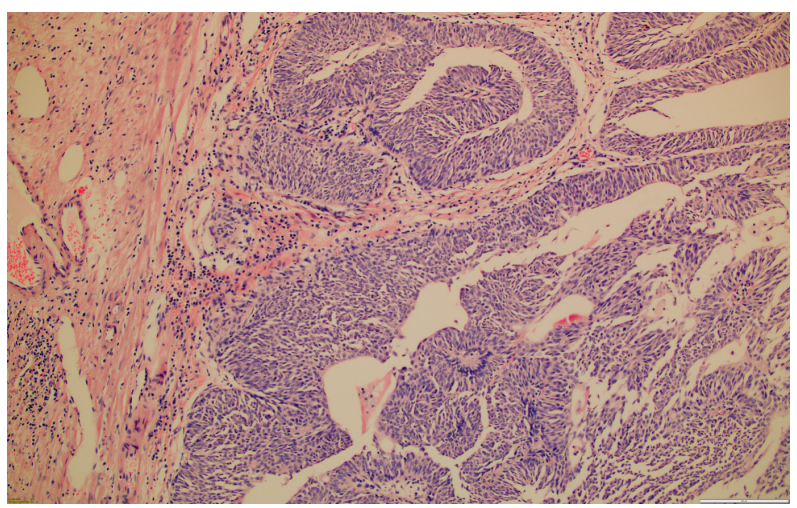

Figure 1. Low grade papillary urothelial carcinoma, 100x, HE.

Case 6: Urothelial papillary carcinoma, high grade, muscle invasion of ureter. Renal parenchyma: moderate chronic inflammation, including subcapsular densification; no significant fibrosis.

Case 7: Urothelial carcinoma, high grade, invasive, solid with divergent differentiation and anaplastic features. There is squamous differentiation with focal keratinisation. Lymphovascular invasion and renal parenchyma invasion. Tumour cells are CK AE1/AE3 and p63 diffusely positive (Figure 6), GATA3 is positive only in the upper (superficial) areas of the tumour, just beneath the urothelium; CK7, Vimentin and PAX8 are negative. This immune profile indicates the urothelial origin of the tumour (GATA3), with squamous and anaplastic transformation (GATA3 loss in poorly differentiated areas).

Case 8: Urothelial carcinoma, high grade, solid, focally with clear cells, invasive, with muscle invasion; large area of necrosis with focal calcification. IHC is confirming the urothelial origin: tumour cells are diffusely and intensely GATA3 positive (Figure 7) and PAX8 negative. Renal parenchyma: diffuse chronic inflammatory infiltrate, minimal fibrosis.

\section{Discussion}

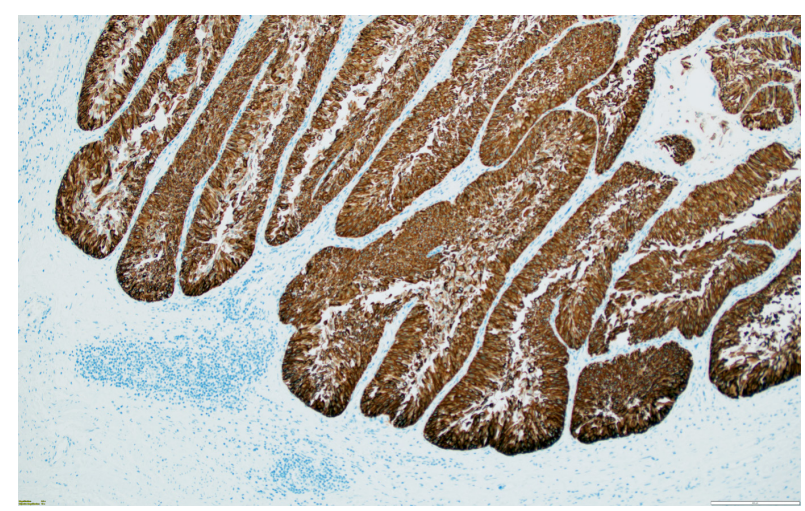

Figure 2. Low grade papillary urothelial carcinoma, inverted, CK7 positive, 100x.100x, SMA.

The study presents new detail of tumour histology, augmented by IHC where necessary to establish the urothelial origin of solid, invasive forms of carcinoma, e.g. discriminating from renal cell carcinoma. It illustrates a stage during development of Balkan endemic nephropathy for patients whose condition required surgical intervention for urothelial cancer.

To summarise the findings (Table 1), renal pelvis tumours from eight BEN subjects were all urothelial carcinomas, graded as per the most recent World Health Organization classification system [16] as low or high grade, as follows:

- two low grade papillary urothelial carcinomas, showing no invasion in the lamina propria or muscularis propria.

- one case with foci of high grade urothelial papillary carcinoma with lamina propria invasion, on a background of low grade urothelial carcinoma; no muscle invasion.

- three high grade papillary urothelial carcinomas, showing invasion of either lamina propria or muscularis propria.

- two cases of high grade urothelial carcinomas, with solid architecture and invasive morphological features.

Consideration also of cortex of the adjoining kidney allowed sight of underlying renal disease, the residual renal parenchyma is

Table 1. Summary of key histopathological characteristics of tumour and kidney in BEN cases.

Case Tumour Kidney

1 LG papillary UC

2 LG papillary UC, no muscle invasion

$3 \quad$ Foci of HG papillary UC with lamina propria invasion on a LG UC background

$4 \quad$ HG papillary UC, muscle invasion

$5 \quad$ HG papillary UC, lamina propria invasion

$6 \quad$ HG papillary UC, muscle invasion

7 HG solid UC with squamous differentiation, vascular invasion

$8 \quad$ HG solid UC, muscle invasion
Mild fibrosis and focal mild chronic inflammation

Mild fibrosis and focal mild chronic inflammation

Mild fibrosis and diffuse severe chronic inflammation

Mild fibrosis and focal mild chronic inflammation

Mild fibrosis and diffuse severe chronic inflammation

Mild fibrosis and zonal moderate chronic inflammation

Not available

Mild fibrosis and diffuse severe chronic inflammation

UC: urothelial carcinoma, HG: high grade, LG: low grade. 


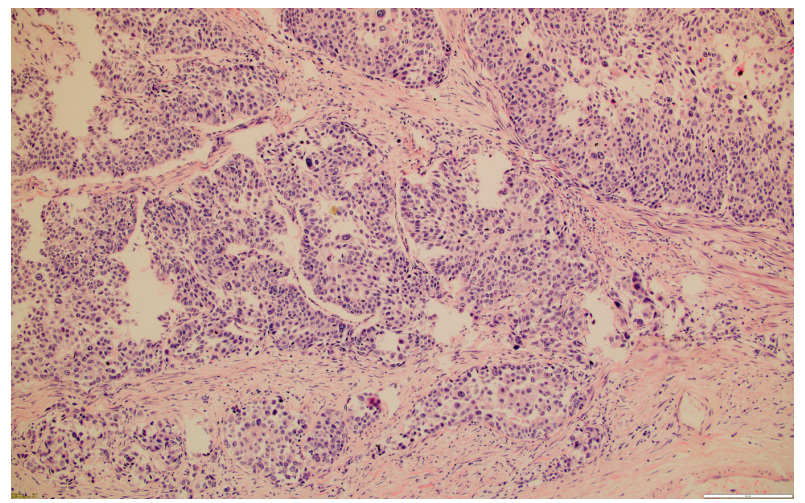

Figure 3. High grade invasive urothelial carcinoma, 100x, HE.

showing in all available cases, mild fibrosis (Figure 8) and variable chronic inflammatory infiltrate (Figure 9), either diffuse or focal. There is no significant relationship between the grade or tumour morphology and the degree of inflammation and/or fibrosis. The consistent renal histological findings characterizing BEN are extensive hypocellular interstitial fibrosis associated with tubular atrophy involving medullary rays, the attending outer medulla and the cortical labyrinth, where it decreases typically from the outer to the inner cortex. Glomerular lesions are associated with periglomerular fibrosis, including ischaemic, microcystic, obsolescent glomeruli, occasional thrombotic microangiopathylike lesions and focal segmental sclerosis-like lesions. Vascular lesions include arteriolar hyalinosis, intimal fibrous hyperplasia and multifocal thickening and splitting of peritubular capillary basement membranes [17].

All renal pelvis tumours were of urothelial origin; IHC confirmed that none had arisen from renal parenchyma, thereby excluding renal cell carcinoma. It appears that, if the tumourigenic agent had generally been in vascular circulation, and then in kidney glomerular filtrate, it only targeted pelvis epithelium without affecting epithelia of the various nephron segments.

A recent study of urothelial carcinomas across Croatia [18] cites historic incidence of upper urothelial tract tumours of the ureter and renal pelvis as 5-10\% of all cases, with notably higher incidence in the Slavonski Brod area where the Balkan nephropathy has been endemic for many years. Half of the upper urothelial cancers $(n=608)$ were in the renal pelvis, both

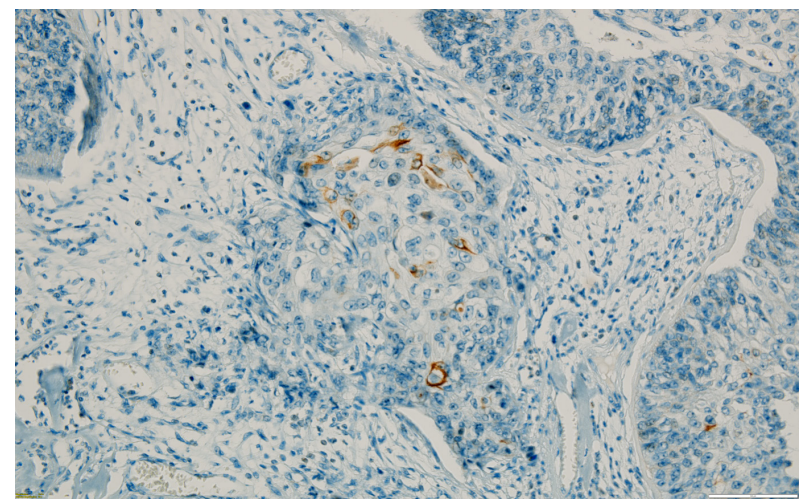

Figure 5. A few CK 20 positive cells in a high grade invasive urothelial carcinoma, 200x.

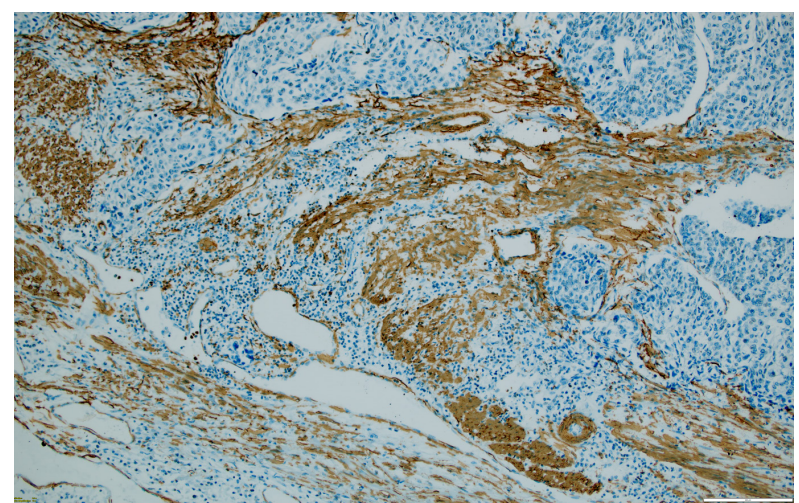

Figure 4. High grade muscle-invasive urothelial carcinoma, as highlighted by smooth muscle actin positive fascicles,

nationally and specifically in the urban area of Zagreb $(n=107)$ and the rural area around Slavonski Brod $(n=86)$. Notably, the river Sava, as a southern geo-political border of Croatia, is artificial concerning hyperendemic BEN which occurs also in Bosnia, and the Republika Srpska further towards Serbia Concurrent findings in Macedonia, where BEN has never been recognised, were $16 \%$ of transitional cell carcinomas among 755 cases of upper urinary tract tumours during 2010-2011, diagnosed in Skopje ( 100 km south of Leskovac) but sourced from across the country [19].

For another Eastern Europe country where BEN has not been recognised, an immunohistochemical study in 2009 of urothelial neoplasms in Slovakia (about $400 \mathrm{Km}$ north of the Croatian BEN area) [20] found that CK7 immuno-staining was positive in all papillary carcinomas. CK 20 was mostly positive $(73.5 \%)$ in urothelial carcinomas. p63 was a diagnostic marker in highgrade urothelial carcinoma, demonstrating micro-invasion of lamina propria, but was less reliable for invasive low-differentiated urothelial carcinomas.

Irrespective of grade, most papillary urothelial carcinomas do not require immunohistochemical analyses for diagnosis. Morphological features are usually self sufficient for this, even the particular forms can be confidently called urothelial. The typical immune profile is: GATA3 and p63 nuclear positive staining, CK7 cytoplasmic positive staining with membranous accentuation, CK20 variable staining, usually significantly less than CK7. PAX8 was constantly negative, excluding thus the renal cell origin (renal cell carcinoma).

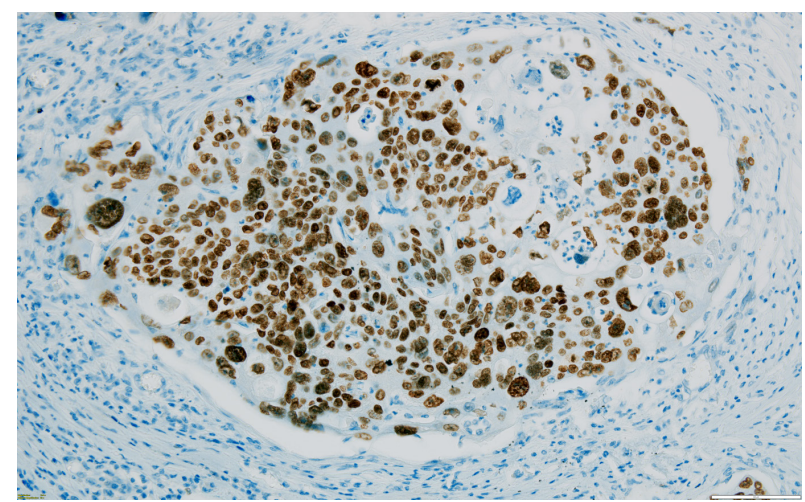

Figure 6. Most cells are positive for p63 in this high grade invasive urothelial carcinoma with squamous features, $200 x$. 


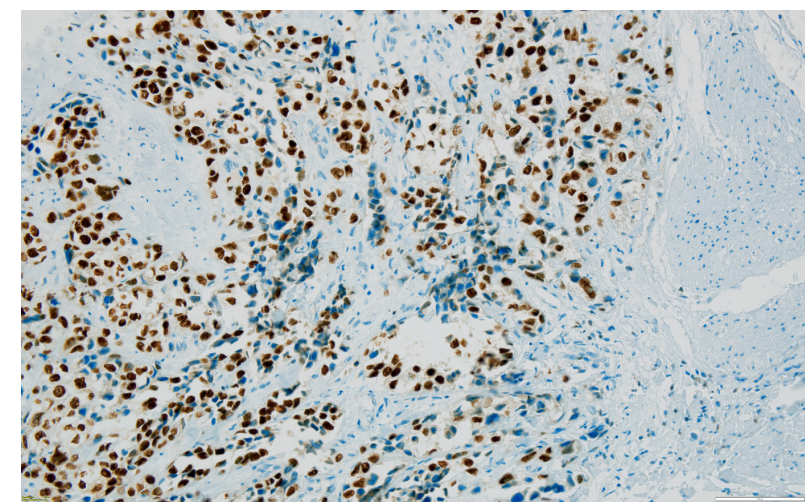

Figure 7. Muscle (on the right side) invasive urothelial carcinoma, high grade, GATA 3 positive nuclei confirming the urothelial origin, 200x.

However, for the two solid renal tumours in our study, IHC was helpful in establishing the urothelial origin:

A) One case is showing divergent differentiation and anaplastic features: squamous differentiation with focal keratinisation is identified. Additionally, lympho-vascular invasion and renal parenchyma invasion is present, certifying the high stage of this tumour. IHC is supportive for the urothelial epithelial origin: the tumour cells are CK AE1/AE3 and p63 diffusely positive; GATA3 is positive, but only in the upper (superficial) areas of the tumour, closer to the urothelium. There is GATA3 loss of expression in divergent and poorly differentiated areas, where CK7, Vimentin and PAX8 are also negative. This overall immune profile indicates the urothelial origin of the tumour, with squamous and anaplastic transformation.

B) The other solid, invasive case has clear cells areas and is showing muscle invasion; there is also a large area of necrosis with focal calcification. IHC is confirming here likewise the urothelial origin: tumour cells are diffusely and intensely GATA3 positive and PAX8 negative, thus excluding a clear cell renal cell carcinoma.

From another point of view, pancytokeratin CK AE1/AE3, GATA3 and p63 gave consistently positive insights and PAX8 was constantly negative. Variable response for CK20 was within a diagnosis of papillary urothelial carcinoma, conforming to the Slovakian study20. CK7 staining was intensely and diffusely

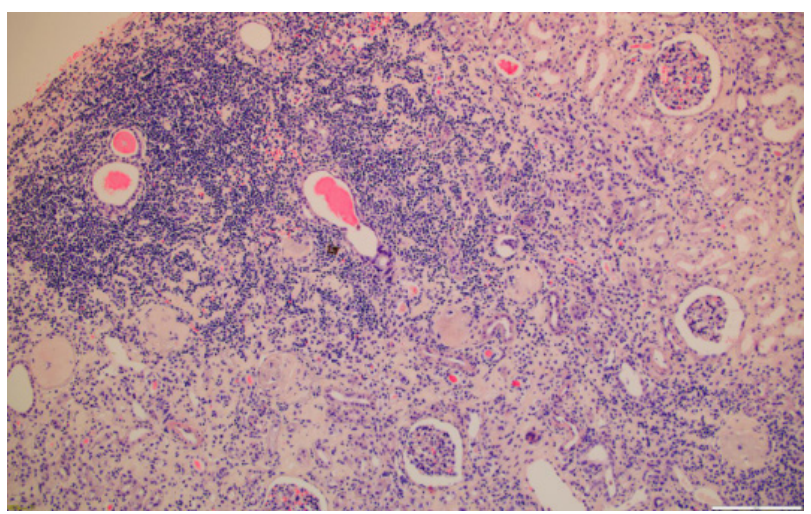

Figure 9. Kidney with severe chronic inflammation, 100x HE.

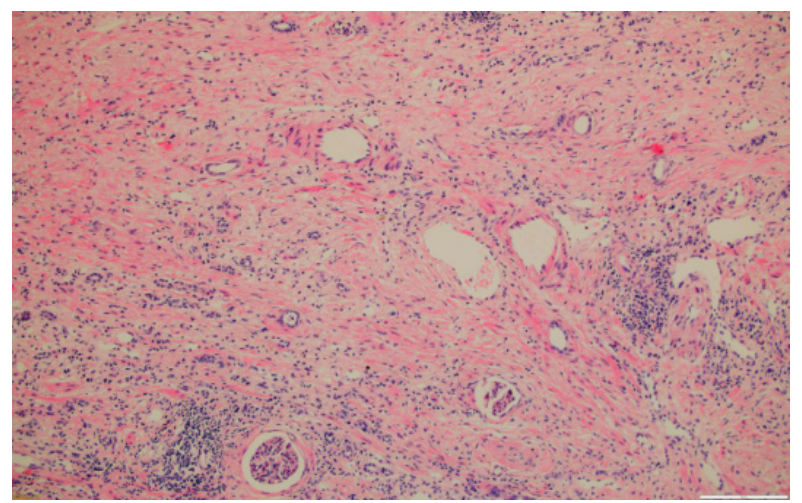

Figure 8. Kidney with severe fibrosis, 100x HE.

positive within papillary urothelial tumours; it contrasted with its absence in the solid urothelial carcinoma with squamous differentiation and anaplastic features. SMA was useful for highlighting invasion of the muscularis propria by tumour; likewise staining for $\mathrm{CD} 34$ for vascular invasion.

Attempts have been made to demonstrate an animal model for aristolochic acid (AA) to mimic the urothelial tumour aspect of the Belgian Chinese herbs nephropathy epidemic in women following a slimming regimen in the early 1990s. Failure to observe this experimentally in rats was followed by a experiment with twelve New Zealand white female rabbits given $0.1 \mathrm{mg} / \mathrm{kg}$ body weight intraperitoneally on five days each week for up to 21 months [21]. Mild toxicities were encountered and interstitial fibrosis was found in kidneys. An in situ lesion, described as a renal cell carcinoma, in two outer-medulla nephrons was noted in one animal at 17 months and a tubulopapillary adenoma in another after 21 months of the continuous administration of AA. Illustration of these tumours was not given; it would be interesting to know their immune profile as within renal parenchyma if archived material exists, although claim of these as sufficient models for human AA tumourigenicity is highly questionable. Also a single urothelial tumour after 450 daily injections into the immediate vicinity of ureters could hardly mimic an oral route as experimental model for urothelial tumours; again the immune profile of that tissue could be interesting.

More recently, following acute per os administration.of AA (50 $\mathrm{mg} / \mathrm{kg}$ ) on 3 consecutive days to female Sprague-Dawley rats [22], moderate temporary morbidity ensued for several days. Six months later, three renal mesenchymal tumours and one oncocytoma were found, but no urothelial lesions occurred in spite of giving a cumulative AA dose about 4-fold greater than in the rabbit study above. Again, to know the immune profile of these tumours may also complement their initial diagnosis, but they do not help to supply positive experimental support for AA in direct causation of BEN-associated urothelial tumours.

The present opportunity to relate tumour histology in BEN urothelial cancer to the renal fibrosis not yet at an end-stage of renal disease, suggests that tumourigenesis is not a response to renal failure. In perceiving a tumourigenic factor, objectivity in its epidemiology is important. Thus, meaningful molecular markers for such need to be perceived in every case of the disease as prescribed for appropriate epidemiological application of Koch's Postulates and the Bradford Hill Criteria as accessibly defined online in Wikipedia. Human exposure would also need to coincide with tumour initiation in sufficient potency and cumulative insult. 
Detection in tumour tissue at a stage probably years or many years later is interesting but inadequate, other than as an indicator of exposure. Historically for BEN, it has been estimated that an individual needs to be resident in an endemic village for at least 20 years [23] to become programmed towards the renal atrophy and its frequently associated urothelial cancer. Concurrently, molecular markers of exposure to AA have been DNA adducts or particular mutational transformations. The former, with long persistence in humans [24] may not be assumed to coincide with tumour initiation which for BEN may occur months or many years prior to detection in tumour. Particular accumulation of such markers can be expected in the metabolic sinks of proliferating cancers in mature adults and can not just be assumed to have initiated a tumour when found following nephrectomy or at death. Notably, AA/DNA adducts have been found in kidney of surgical patients treated for renal cell carcinoma [25], implying exposure to AA via common herbal remedies in Romanian regions in which BEN has not been recognised. Thus exposure is not inevitably an indicator of prospective BEN tumours disease but in the cited study was claimed as evidence of AA as causing renal cell carcinoma instead. Also, it is not clear whether adducts can occur in circulating blood, thereby presenting analytical confusion in vascularised tissues.

A mutational transformation has been claimed as diagnostic for AA exposure [26], but without citing evidence for its exclusivity for AA-derived carcinoma. Subsequently, analysis from endemic village residents in Croatia and Serbia of upper urinary tract cancers $(n=97)$ from unilateral nephroureterectomy recognised AA signatures in only 42 cases [27]; absence from over half of cases fails to provide objective aetiological correlation with AA exposure. Unfortunately however, the studies did not include tumour histopathology. Recently published bibliographic analysis of research trends on aristolochic acids [28] has also concluded that 'there is insufficient epidemiological and animal experimental evidence to support a direct correlation between the mutational signature of AA and cancer'.

Recent application of sensitive chemical analysis has demonstrated plant uptake of AA from A. clematitis debris in soil [29] and occurrence in soil water [30], thereby offering additional prospect of human exposure to traces of AA via agricultural crops. This has not yet been quantified for human exposure; exposure from all sources would need to match the highly mosaic distribution of BEN villages and focally amongst the households within. However, the mosaic arrangement of endemic villages and the mosaic arrangement of families affected by BEN in endemic villages may be influenced by genetic predisposition. Early experimental animal toxicity studies on AA seemed to need daily oral administration in the $\mathrm{mg} / \mathrm{kg}$ body weight range for tumours to arise; these occurred near the point of administration. The long route from mouth to urothelium presents further challenge unless particular sensitivity resides there, but human AA intake matching that estimated for the Belgian nephropathy epidemic would need to be plausible.

For a urinary metabolomic study in Romania in 2004, about 100 miles north of the area of present study area, illustration of A. clematitis in the village of Erghevita [31] resonates with its 1970s consideration as an endemic agricultural weed (known also as Birthwort)6, but that did not seem to correlate closely with BEN's mosaic occurrence. The metabolomic study differentiated cases of nephropathy from controls, but one Romanian control tended towards the BEN cluster. All nephropathy cases had been in late stage renal failure, on regular haemodialysis, and predictably some will have had urothelial carcinoma. Thus the question arose as to whether incipient BEN, and possibly a tumour, could be indicated metabolomically in urine, but ethical considerations limited informal experimentation.

\section{Conclusions}

This study encompasses only a few cases of urothelial carcinomas, but with a wide range of forms, covering the whole spectrum, from the quiescent low grade papillary to the solid, invasive, poorly differentiated carcinoma. There is no evident morphological feature within these tumours which could reflect any of the abovementioned ethiopathogenic mechanisms. Histologically, they look identical to urothelial neoplasms from elsewhere, from any other non-BEN area; the differences might be on a molecular level, waiting to be explored.

The pathology of BEN is characterized by a progressive atrophy and sclerosis of all structures of the kidney, and it shares similarities with tubulointerstitial kidney diseases, being difficult to discriminate between a BEN and a non-BEN nephropathy, especially at an incipient stage. There is no diagnostic feature of BEN, but the set of epidemiological, clinical and biochemical data along with the pattern of pathologic injury can be highly suggestive of this entity.The present illustration of pelvic tumour histology at an intermediate stage of BEN offers opportunity to widen the findings across the Balkans where suitable tissues may be archived, and to relate to pathologies elsewhere. It offers closer comparison with human urothelial tumours closely associated with herbal remedies and medications.

\section{Ethical policy}

The research was approved by the Ethics Committee of the General Hospital Leskovac, Serbia on 16-11-2018. No. 11550/2.

\section{Author contributions}

In a close informal and unfunded collaboration, PM and DH proposed the project, obtained and interpreted histopathology data and drafted the manuscript. NP and GS selected archived tissues of all relevant subjects from within their hyperendemic area and provided histology sections.

\section{Competing interests}

All authors declare no competing interests.

\section{Funding}

This research did not receive any specific grant from funding agencies in the public, commercial, or not-for-profit sectors.

\section{Acknowledgements}

Assistance in sourcing archived material by Dr Ivana Davinic, Clinic of Nephrology, Medical Faculty, Nis, and Dr Jelena Stanojevic, Institute of Pathology, Medical Faculty, Belgrade is gratefully acknowledged.

\section{References}

1. Tanchev Y, Dorossiev D: The first clinical description of Balkan endemic nephropathy (1956) and its validity 35 years later. In Mycotoxins, Endemic Nephropathy and Urinary Tract Tumours Ed. Castegnaro M, Plesina R, Dirheimer G, Chernozemsky I.N. Bartsch $\mathrm{H}$ International Agency for Research on Cancer, Lyon..Scientific Publication 115. 1991. p.21-28.

2. Chernozemsky IN, Stoyanov IS, Petkova-Bocharova TK, Nikolov IG, Draganov IV et al: Geographic correlation between the occurrence of endemic nephropathy and urinary tract tumours in Vratza district, Bulgaria Intl J Cancer 1977, 19: 1-11. 
3. Petkovic SD: Epidemiology and treatment of renal and uretera cancer. J Urol 1975,114: 858-865.

4. Radovanovic Z: Epidemiological characteristics of Balkan endemic nephropathy in eastern regions of Yugoslavia. In Mycotoxins, Endemic Nephropathy and Urinary Tract Tumours Ed. Castegnaro M, Plesina R, Dirheimer G, Chernozemsky I.N. Bartsch H International Agency for Research on Cancer, Lyon..Scientific Publication 115. 1991. p.11-20.

5. Sostaric B, Vukelic M: Characteristics of urinary tract tumours in the area of Balkan endemic nephropathy in Croatia, In Mycotoxins, Endemic Nephropathy and Urinary Tract Tumours Ed. Castegnaro M, Plesina R, Dirheimer G, Chernozemsky I.N. Bartsch H International Agency for Research on Cancer, Lyon..Scientific Publication 115. 1991. p.29-35.

6. Austwick PK: Balkan nephropathy. J Roy Soc Med 1975, 68: 219221.

7. Barnes JM, Carter RL, Periarianis GC, Austwick, PKC, Flynn FV, Aldridge WN: Balkan (endemic) nephropathy and a toxin-producing strain of Penicillium verrucosum var cyclopium, an experimental model in rats. Lancet 1977, 289; 671-675.

8. Boorman, GA: Toxicology and carcinogenesis studies of ochratoxin A (CAS No. 303-47-9) in F344/N rats (gavage studies). Natl Toxicol Program Tech Rep. 1989, 358: 1-142.

9. Bendele AM, Carlton WW, Krogh P, Lillehoj EB: Ochratoxin A carcinogenesis in the $(\mathrm{C} 57 \mathrm{BL} / 6 \mathrm{~J} \mathrm{X} \mathrm{C} 3 \mathrm{H}) \mathrm{F} 1$ mouse J Natl Cancer Inst. 1985, 75: 733-742.

10. Nortier JL, Martinez M-CM, Schmeiser HH, Arlt VM, Bieler CA, Petein M, Depierruex MF, DePauw L, Abramowicz D, Vereerstraeten P, Vanherweghem J-L: Urothelial Carcinoma Associated with the Use of a Chinese Herb (Aristolochia fangchi). N Engl J Med 2000, 342:1686-1692.

11. Pavlovic NM: A historical overview of Balkan Endemic Nephropathy (BEN) in relation to published hypotheses. Pril (Makedon Akad Nauk Umet Odd Med Nauki) 2014,35: 71-79.

12. Herman D, Mantle P: Immunohistochemical analysis of rat renal tumours caused by ochratoxin A. Toxins 2017, 9: 384.

13. Herman D, Mantle P: Immunohistochemical review of Leydig cell lesions in ochratoxin A-treated Fischer rats and controls. Toxins 2019, 11: 480 .

14. Castegnaro M, Plesina R, Dirheimer G, Chernozemsky IN, Bartsch H: Eds. Mycotoxins, Endemic Nephropathy and Urinary Tract Tumours International Agency for Research on Cancer, Lyon. .Scientific Publication 115. 1991.

15. Jelakovic B, Dika Z, Arlt VM, Stiborova M, Pavlovic NM, Nikolic J, Colet J-M, Vanherweghem J-L, Nortier JL: Balkan endemic nephropathy and the causative role of aristolochic acid. Seminars in Nephropathy 2019, 39: 284-296.

16. Moch H, Humphrey PA, Ulbright TM, Reuter VE: WHO Classification of Tumours of the Urinary System and Male Genital System, 4th Edition, WHO Press, 2016, 2: 77-108.

17. Pavlovic NM: Balkan endemic nephropathy - current status and future perspectives. Clin Kidney J 2013, 6: 257-265.

18. Medunjarin D, Sonicki Z, Vena JE, Cvitkovic A, Robb SW: Geographic distribution and risk of upper urothelial carcinomas in Croatia, 2001-2011. BMC Cancer 2019,19:950 doi: 10.1186/s12885019-6160-9

19. Kostadinova-Kunovska S, Janevska V, Komina S, Dukova B, Bogdanovska-Todorovska $\mathrm{M}$ et al: Incidence of urinary tract tumours in a two-year period (2010-2011) at the Institute of Pathology, Faculty of Medicine, Skopje, Macedonia. Contributions Sec Med Sci. 2014, XXXV 1, 65-70.

20. Rajcani J, Kajo K, Adamkov M, Moravekova E, Lauko L, Felcanova D, Bencat M: Immunohistochemical characterization of urothelial carcinoma. Bratislava Med J 2013, 114: 431-438.

21. Cosyns J-P, Dehoux J-P, Guiot Y, Goebbels R_M, Robert A, Bernard AM, van Yperselle de Strhiou C: Chronic aristolochic acid acid toxicity in rabbits: A model of Chinese herbs nephropathy. Kidney International 2001, 59: 2164-2173.

22. Cui M, Liu Z-H, Qui Q, Li H, Li L-S: Tumour induction in rats following exposure to short-term high-dose aristolochic acid 1. Mutagenesis 2005, 20: 45-49.

23. Ceovic S, Hrabar A, Radonic M: An etiological approach to Balkan endemic nephropathy based on the investigation of two genetically different populations. Nephron 1985, 40: 175-179.

24. Schmeiser HH, Nortier JL, Singh R, Gamboa da Costa G, Sennesael J, Cassuto-Viguier E, Ambrosetti D, Rorive S, Pozdzik A et al: Exceptionally long-term persistence of DNA adducts formed by carcinogenic aristolochic acid $\mathrm{I}$ in renal tissue from patients with aristolochic acid nephropathy. Int J Cancer 2014, 135: 502-507.

25. Turesky RJ, Yun BH, Brennan P, Mates D, Jinga V et al: Aristolochic acid exposure in Romania and implications for renal cell carcinoma. Brit J Cancer 2015, DOI:10.1038/bjc.2015.402.

26. Grollman AP, Shibutani S, Moriya M, Miller F, Wu L, Moll U, Suzuki N, Fernandes A, Rosenquist T, Medverec Z et al: Aristolochic acid and the etiology of endemic (Balkan) nephropathy. Proc Natl Acad Sci USA 2007, 104: 2129-2134.

27. Moriya M, Slade N, Brdar B, Medverec Z, Tomic K, Jelakovic B, Wu L, Truong S, Fernandes A, Grollman AP: TP53 Mutational signature for aristolochic acid: an environmental carcinogen. Int $\mathrm{J}$ Cancer 2011,129: 1532-1536.

28. Zhou Q, Pei J, Poon J, Lau AY, Zhang L, Wang Y, et al: Worldwide research trends on aristolochic acids (1957-2017): Suggestions for researchers. PLoS ONE 2019,14: e0216135. https://doi.org/10.1371/ journal.pone.0216135.

29. Li W, Chan C-K, Liu Y, Yao J, Mitic B, Kostic EN, Milosavlievic B, Davinic I, Orem WH, Tatu CA et al: Aristolochic Acids as Persistent Soil Pollutants: Determination of Risk for Human Exposure and Nephropathy from Plant Uptake J Agric Food Chem 2018, 66: 1146811476.

30. Ka-Ki Tung K-K, Chan C-K, Zhao Y, Chan K-KJ, Liu G, Pavlovic NM, Wan Chan W: Occurrence and Environmental Stability of Aristolochic Acids in Groundwater Collected from Serbia: Links to Human Exposure and Balkan Endemic Nephropathy. Environ Sci Technol 2020, 54: 1554-1561.

31. Mantle P, Modalca M, Nicholls A, Tatu C, Tatu D, Toncheva D: Comparative 1HNMR metabolomic urinalysis of people diagnosed with Balkan endemic nephropathy, and healthy subjects, in Romania and Bulgaria: a pilot study, Toxins 2011, 3: 815-833. 